\title{
Right axillary artery conduit for antegrade deployment of a thoracic aortic endoprosthesis
}

\author{
James H. Wudel, MD, ${ }^{a}$ and James B. Williams, MD, ${ }^{b}$ Lincoln, Neb, and Peoria, III
}

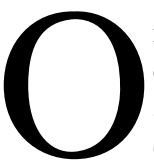

ne of the ongoing challenges associated with the size of devices used in thoracic aortic endografting is arterial access. Intraoperative conduits are frequently required, ${ }^{1}$ or patients are excluded from endovascular repair because their anatomy precludes delivery of the device. ${ }^{2}$ Artery size, artery tortuosity, and previous aortic stent grafting all may complicate the retrograde endovascular navigation required to reach the target site safely. As an alternative, we describe the technique of placing a prosthetic vascular conduit on the right axillary artery to facilitate antegrade deployment of a thoracic endograft in a patient with an enlarging descending thoracic aneurysm, multiple comorbidities, and a previously placed infrarenal endograft.

\section{Clinical Summary}

A 79-year-old woman was seen with an enlarging descending thoracic aortic aneurysm measuring $7.5 \mathrm{~cm}$. She had a history of hypertension, obesity, and an abdominal aortic bifurcated endograft (Gore Excluder; W. L. Gore \& Associates, Inc, Flagstaff, Ariz). Preoperative aortography showed a type $\mathrm{C}$ arch without extracranial great vessel disease. Multiplanar computed tomography showed a suitably placed abdominal endograft within tortuous aorta and iliac arteries (Figure 1). Her anatomy was considered suboptimal for retrograde deployment because of the iliac vessel size and tortuosity and because of the previous abdominal endograft. Antegrade deployment through a right axillary conduit was therefore performed.

With the patient under general anesthesia after systemic heparinization, a 10-mm diameter, 20 -cm long prosthetic vascular graft was sutured as a conduit to the right axillary artery through an infraclavicular incision. With the vascular conduit perfused and clamped, distal access through the conduit was obtained, and the origin of the head vessels was subsequently confirmed on arteriography. Percutaneous bilateral common femoral artery access was obtained. After placement of an access sheath through the Dacron polyester fabric conduit into the descending aorta, a 0.035-inch wire was

From Nebraska Heart Institute, Lincoln, Neb, ${ }^{\mathrm{a}}$ and Cardiac Thoracic and Endovascular Therapies, Peoria, Ill. ${ }^{\text {b }}$

JBW reports consulting fees from W. L. Gore \& Associates, Inc, Flagstaff, Ariz; from Boston Scientific Corporation, Boston, Mass; and from Endologix, Irvine, Calif. JBW also reports grant support from Cordis Corporation, Miami Lakes, Fla.

Received for publication July 31, 2007; accepted for publication Sept 18, 2007.

Address for reprints: James H. Wudel, MD, Nebraska Heart Institute, Lincoln, NE 68506 (E-mail: jwudel@neheart.com).

J Thorac Cardiovasc Surg 2008;135:436-7

$0022-5223 / \$ 34.00$

Copyright (C) 2008 by The American Association for Thoracic Surgery doi:10.1016/j.jtcvs.2007.09.059

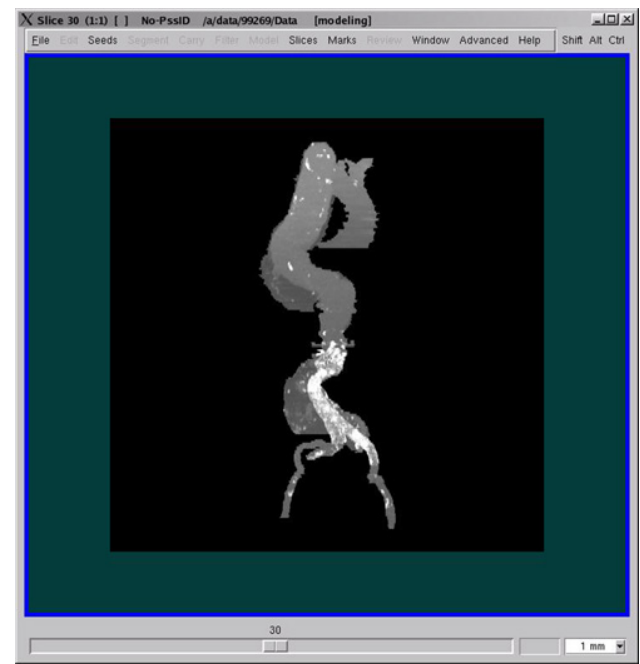

Figure 1. Multiplanar reconstruction showing descending aneurysm, vascular tortuosity, and previous endograft.

passed through the Dacron conduit, snared from below, and brought out externally through the right femoral sheath. A JB1 SlipCath (Cook Medical Inc, Bloomington, Ind) was then passed through and through antegrade over the 0.0035 wire to allow exchange for a Lunderquist (Cook Medical) wire. The Gore TAG 24F sheath (W. L. Gore) was placed through the external graft by the Lunderquist wire, with the sheath stopping just distal to the vertebral and right carotid artery origins.

Gore TAG grafting was then performed antegrade from just distal to the subclavian artery to just proximal to the celiac artery with a total of three overlapping endoprostheses (Figure 2). Completion arteriography showed no endovascular leakage. The vascular graft was amputated and oversewn near its base. The patient awakened without any neurologic deficit and was discharged 5 days later. Two months after the operation, she continues to do well, with postprocedure computed tomography showing no clinical abnormalities.

\section{Discussion}

The relatively large diameter of the thoracic aorta and the technical limits of catheter-mounted endografts make vascular access a critical part of the preoperative evaluation in stent graft candidates. Small iliac vessel size and tortuosity complicate the manipulation of a large sheath retrograde to the thoracic landing zone. In a recent series of 126 patients undergoing thoracic stent graft evaluation, inadequate vascular access accounted for nearly a third of the anatomic exclusions. ${ }^{2}$ In the first pivotal trial of an approved device in the United States, $15 \%$ of patients required an intraoperative 

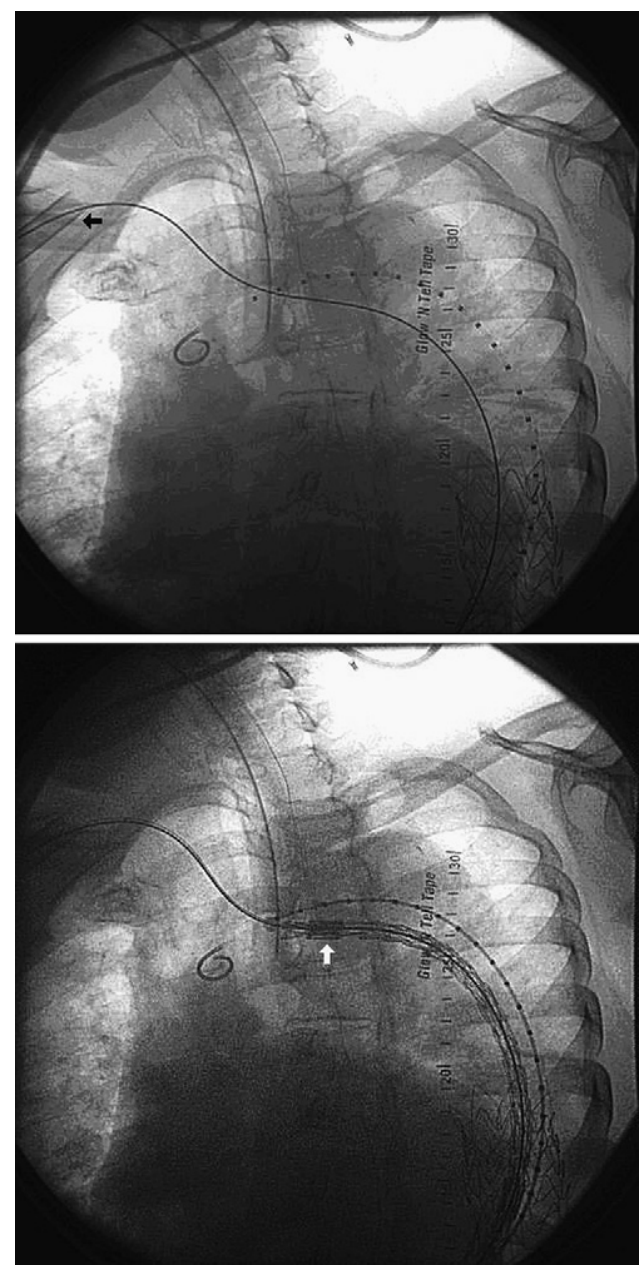

Figure 2. Left anterior oblique projection of endograft sheath positioned in distal right axillary artery (black arrow, above) and improved endograft trackability through antegrade approach (white arrow, below). access conduit. ${ }^{1}$ In addition, navigating a large sheath retrograde has the potential to disrupt a previously placed abdominal stent graft. All these factors contributed to the evaluation of an alternate access site in this case.

Access sites for deployment of thoracic endografts through either common carotid artery have been reported. ${ }^{3-5}$ Potential advantages of using the right axillary artery with a conduit include (1) direct avoidance of repeated manipulation of the carotid artery, with its potential for vessel injury, atheroembolism, and cerebral hypoperfusion, (2) ability of the extracorporeal graft to cover most of endograft sheath length and allow a lower intravascular device profile, and (3) provision by the right axillary femoral wire of improved trackability for an antegrade delivery through disadvantaged iliac arteries. Suitable arch anatomy must be confirmed preoperatively for this approach. In addition, this approach may not be suitable for other thoracic device models without modification

Thoracic endografting continues to evolve and is being applied for increasingly complex cases. Accessory access sites and adjunctive maneuvers in these cases are vital. The appreciation that brachiocephalic vessels may be used for access will facilitate the use of these devices in the treatment of diseases involving not only the descending thoracic aorta but also the ascending aorta, root, and aortic valve.

\section{References}

1. Cho JS, Haider SE, Makaroun MS. Endovascular therapy of thoracic aneurysms: Gore TAG trial results. Semin Vasc Surg. 2006;19:18-24.

2. Jackson BM, Carpenter JP, Fairman RM, Moser GW, Pochettino A, Woo EY, et al. Anatomic exclusion from endovascular repair of thoracic aneurysm. J Vasc Surg. 2007;45:662-6.

3. Murray D, Ghosh J, Khwaja N, Murphy MO, Baguneid MS, Walker MG. Access for endovascular aneurysm repair. $J$ Endovasc Ther. 2006;13: 754-61.

4. Heidenrich MJ, Neschis DG, Costanza MJ, Flinn WR. Endovascular repair of a penetrating thoracic aortic ulcer by way of the carotid artery. $J$ Vasc Surg. 2003;38:1407-10.

5. Murray D, Ghosh J, Khwaja N, Murphy MO, Baguneid MS, Walker MG. Staged open and endovascular repair of thoracoabdominal aneurysms using the common carotid artery. Ann Vasc Surg. 2005;19: 103-7. 